\title{
COVID-19 e sistema imune: qual o papel do exercício físico e recomendações práticas para adultos?
}

\author{
COVID-19 and immune system: what is the role of physical \\ exercise and practical recommendations for adults?
}

Resumo A pandemia global de COVID-19 trouxe inúmeros desafios para a humanidade. O COVID-19 engatilha um agressivo processo inflamatório em alguns indivíduos, denominado de "tempestade de citocinas", desregulando o sistema imunológico e favorecendo o acometimento de órgãos e, às vezes, resultando em complicações mais graves, como a síndrome respiratória aguda grave. Em tempos de isolamento social, a inatividade física pode afligir a população; no entanto, uma parcela dos indivíduos está se exercitando em domicílio. Evidências demonstram que o exercício físico agudo aeróbio e de moderada intensidade até 60 minutos favorece a manutenção de um sistema imunológico saudável, enquanto que exercícios extenuantes predispõem o risco de infecção do trato respiratório superior (ITRS). Já o exercício físico regular promove um ambiente anti-inflamatório, essencial para o combate de inúmeras comorbidades e o envelhecimento precoce. Portanto, o exercício físico de intensidade, volume e duração controlados e realizados com segurança promovem um ambiente anti-inflamatório favorável, embora sejam necessários estudos para verificar se existe essa proteção contra o COVID-19. Assim, o objetivo deste artigo de opinião é discutir a imunologia do exercício e sua possível proteção contra o COVID-19 e guiar recomendações práticas de exercícios físicos que possam ser feitas no próprio domicílio.

Palavras-chave: Imunologia do Exercício. Pandemia. RecoMENDAÇÕES.

Aвstract The global pandemic of COVID-19 has brought countless challenges to humanity. COVID-19 triggers an aggressive inflammatory process in some individuals called a "cytokine storm", deregulating the immune system and favoring organ damage and serious complications, such as severe acute respiratory syndrome. In times of social isolation, physical inactivity can afflict the population; however, a portion of individuals are exercising at home. Evidence shows that acute aerobic exercise of moderate intensity up to 60 minutes favors the maintenance of a healthy immune system, while strenuous exercise predisposes the risk of upper respiratory tract infection (URTI). Regular physical exercise, on the other hand, promotes an anti-inflammatory environment, essential for combating numerous co-morbidities and premature aging. Therefore, physical exercise of controlled intensity, volume, and duration, safely performed, promotes a favorable anti-inflammatory envi-

André Katayama Yamada Luiz Otávio Barreto Polis ${ }^{1}$ ${ }^{\mathrm{I}}$ Universidade Metodista de Piracicaba (UNIMEP), Piracicaba/SP, Brasil ronment, although studies are needed to verify whether this protection against COVID-19 exists. Thus, the objective of this opinion article is to discuss the immunology of exercise and its possible protection against COVID-19 and to guide practical recommendations for physical exercises that can be performed at home.

KeyWORdS: Exercise immunology. Pandemy. Recommendations. 


\section{INTRODUÇÃO}

O mundo atual vive uma pandemia do novo coronavírus, levando a desafios econômicos, sociais e de saúde pública pouco vistos na história da humanidade. A doença do coronavírus 2019, originada na China, foi denominada de COVID-19 e foi reconhecida como pandemia pela Organização Mundial de Saúde (OMS) no dia 11 de março. ${ }^{1} \mathrm{O}$ COVID-19 é altamente contagioso e alguns indivíduos podem desenvolver a forma grave da doença acompanhada de síndrome respiratória aguda grave, necessitando de suporte de oxigênio. ${ }^{2}$ É transmitida principalmente por gotículas respiratórias expelidas no ar (geralmente tosses e espirros) por indivíduos infectados e até mesmo por fezes. ${ }^{2} \mathrm{~A}$ replicação viral primária ocorre no epitélio da mucosa do trato respiratório superior (cavidade nasal e faringe). ${ }^{1}$ Clinicamente, em alguns casos, se observa uma "tempestade inflamatória" intensa durante a infecção, resultando em inflamação pulmonar descontrolada, predispondo os pacientes a um quadro fatal. ${ }^{3}$ Estudo com 41 pacientes com COVID-19 em estado severo e hospitalizados demonstrou que ocorre elevação de citocinas IL-2, IL-7, IL-10, G-CSF, IP-10, MCP-1, MIP-1A e TNF- $\alpha .{ }^{4}$ Existem apenas informações limitadas no status de sistema imune inato de pacientes com COVID-19. No entanto, já foram observados aumentos nos neutrófilos totais (38\%), redução no total de linfócitos (35\%), aumentos de IL-6 (52\%) e aumento de proteína-C reativa (84\%). ${ }^{5}$ Assim, percebe-se um agressivo processo inflamatório nos pacientes com COVID-19.

Tendo em vista esse cenário de imunossupressão em pacientes acometidos por CO-
VID-19, será que o exercício físico regular prévio (indivíduos ativos) poderia ter um efeito anti-inflamatório e protetor ao indivíduo? Um acumulado corpo de evidência demonstra que o exercício físico com uma dose-resposta adequada pode sim amplificar o sistema imune, e que será abordado neste artigo de opinião nos próximos parágrafos.

\section{EXERCÍCIO FÍSICO E SISTEMA IMUNE}

O exercício físico tem um profundo impacto nas células imunológicas inatas. Por exemplo, durante uma sessão de exercício físico, células natural killers (NK) viajam na corrente sanguínea em vasto número. ${ }^{6}$ Após o exercício, essas células migram para os sítios de inflamação para rastrear patógenos e células danificadas. ${ }^{7}$ Esse processo também auxilia o sistema imune a detectar células cancerígenas. ${ }^{8}$ Outra linha de defesa é a imunidade adaptativa, composta basicamente de células de linfócitos B e T. Exercício físico regular por décadas mantém saudável a população de linfócitos $\mathrm{T}$ imaturos no processo de envelhecimento, o que leva o sistema imune a melhor identificar patógenos e câncer na senilidade. ${ }^{9}$

Sessões agudas de exercícios físicos aeróbios de intensidade moderada a vigorosa de até 60 minutos de duração resultam em atividade antipatogênica dos macrófagos teciduais em paralela a aumentada recirculação de imunoglobulinas, citocinas anti-inflamatórias, neutrófilos, células NK, células T citotóxicas e células B imaturas, que exercem papéis cruciais na atividade de defesa do sistema imune. ${ }^{10,11,12,13}$ Nesses exercícios de curta duração, hormônios do estresse e 
citocinas pró-inflamatórias não se elevam consideravelmente. ${ }^{14}$ Já para exercícios físicos extenuantes, diversas alterações em vários parâmetros imunológicos são observadas, predispondo à imunossupressão. ${ }^{15}$ Por exemplo, existem dramáticas diferenças nas respostas imunes em indivíduos que realizaram maratona ${ }^{16}$ quando comparados a indivíduos que fizeram caminhada. ${ }^{10}$ Dados preliminares também sugerem que a capacidade metabólica da célula imune se apresenta diminuída durante a recuperação de sessões de esforços intensos, resultando em disfunção imunológica transitória. ${ }^{16}$ Assim, em situações de isolamento social a qual vivemos recomenda-se atividades de até 60 minutos de intensidade moderada, visando aumentar a imunidade.

Mas será que exercícios extenuantes podem levar à sobrecarga imunológica e resultar em doença? Apesar de os estudos serem controversos, evidências mais clássicas disponíveis apontam que sim. No entanto, os pesquisadores questionam se o exercício físico per si só seria responsável diretamente por infecções do trato respiratório superior (ITRS). ${ }^{17}$ Estudos epidemiológicos indicam que atletas engajados em provas de maratona e ultramaratona ou treinamento intenso apresentam maior risco de desenvolver ITRS. ${ }^{18} \mathrm{O}$ risco de adoecer aumenta ainda mais quando os indivíduos não descansam o suficiente em sessões de treinamento intenso e são expostos a estressores como falta de sono ou estresse mental, ${ }^{19}$ desregulando o sistema imune, o que tem implicação significativa no isolamento social. No entanto, o mais provável é que um quadro de imunossupressão causada pelo esforço não aconteça, pois se acredita que a maioria das pessoas esteja exercitando em intensidade e frequência mais baixas do que de costume nestes períodos de confinamento.

Cada sessão de exercício físico moderado promove aumento transitório de imunossegurança e quando repetido por semanas ou meses promove um efeito anti-inflamatório sistêmico e tecidual. ${ }^{20} \mathrm{Um}$ acumulado de evidências aponta que o exercício físico promove um potente efeito anti-inflamatório, diminuindo a inflamação em diversas comorbidades como diabetes, hipertensão, obesidade e no envelhecimento. ${ }^{21,22,23}$ Isto tem grande implicação para a proteção contra o COVID-19, pois a maioria dos casos fatais ocorre nessa população com doenças crônicas e idosos. ${ }^{24,25}$ No entanto, são necessários estudos, pois não há evidências de que o efeito do treino prévio proteja contra o COVID-19 nesses grupos de risco.

Estudos epidemiológicos sugerem que o exercício físico regular está associado com menor mortalidade e taxa de incidência para influenza e pneumonia. ${ }^{26,27}$ Em estudos experimentais, exercício físico aeróbio moderado (30 $\mathrm{min} / \mathrm{dia}$ ) reduziu a mortalidade de camundongos pela influenza. ${ }^{28}$ No entanto, camundongos que se exercitaram por longa duração ( 2.5 h/dia) exibiram aumento de morbidade, mas não houve diferenças estatisticamente significantes na mortalidade quando comparados a camundongos sedentários. ${ }^{28}$ Indivíduos idosos engajados em exercícios aeróbios regulares (10 meses) tendem a ter uma melhor resposta dos anticorpos quando imunizados pela influenza. ${ }^{29}$ Astronautas que apresentavam maior aptidão cardiorrespiratória pré-expedição e condicionamento muscular esquelético anterior 
a uma missão de seis meses na Estação Espacial Internacional eram menos propensos a reativar viroses da herpes durante a missão. ${ }^{30}$ Cópias de DNA viral da Epstein-Barr apresentavam mais baixos nos astronautas condicionados, indicando habilidade reduzida em infectar outras pessoas. Esses dados em conjunto demonstram claramente que o exercício físico facilita a proteção imunológica da vacinação contra vírus.

Adicionalmente, pesquisas têm demonstrado que a cicatrização cutânea é mais acelerada em indivíduos que se exercitam regularmente quando comparadas a indivíduos sedentários. ${ }^{31}$ Cicatrização cutânea acelerada reduz o risco de entrada de bactérias e vírus em pessoas ativas.

\section{CONSIDERAÇÕES E RECOMENDA- ÇÕES PRÁTICAS EMBASADAS EM EXPERIÊNCIAS PROFISSIONAIS}

Devido à pandemia, como alternativa a exercícios físicos ao ar livre, em academias, parques e ruas, as pessoas estão se exercitando em domicílio. No entanto, é preciso certo cuidado e conhecimento, que serão abordados nos parágrafos a seguir.

Quando se fala em prevenção e combate a algumas doenças e, ao mesmo tempo, no sentido da promoção da "saúde" e do "bem-estar geral", todo indivíduo deveria procurar realizar semanalmente, de forma sistemática e planejada, três principais tipos de atividades físicas: exercícios aeróbicos (cardiorrespiratórios), de força (neuromuscular) e de alongamento, o que certamente traria inúmeros benefícios, vantagens e resultados positivos para o organismo como um todo. ${ }^{32,33}$ Classicamente, o primeiro contribui para o aumento da capacidade cardiorrespiratória, maior gasto calórico, ganho de resistência, e condicionamento físico; ${ }^{34,35}$ o segundo, leva à hipertrofia dos mais variados grupos musculares, podendo implicar no ganho de força, prevenção de quedas, maior controle postural, ${ }^{35,36}$ e o terceiro, de grande relevância para redução de lesões, dores e aumento da flexibilidade. ${ }^{37}$ Desse modo, pode-se constatar que cada uma das três atividades supracitadas, tendo em vista as suas características e finalidades, tem relação direta com as questões de saúde e bem-estar. Como já citado, exercícios físicos regulares que relaxam a musculatura e a mente são também importantes neste período para fortalecer o sistema imunológico. E, portanto, se trabalhados em conjunto, pelos mais variados profissionais, ou se praticados em sua completude pela comunidade, normalmente, alcançariam resultados ainda mais surpreendentes e expressivos nesse viés e no combate às enfermidades e doenças em geral.

A mais recente recomendação de atividade física para adultos foi estabelecida pelo Colégio Americano de Medicina do Esporte. ${ }^{38}$ Dessa forma, recomenda-se exercícios de 30 minutos diários durante cinco dias na semana, totalizando 150 minutos por semana de atividade aeróbia (exercícios cardiorrespiratórios) de intensidade moderada/vigorosa, 75 minutos por semana de atividade aeróbia vigorosa, ou a combinação delas, com a meta de atingir um gasto energético equivalente $\mathrm{a} \geq 500$-1000 MET minutos por semana, visando benefícios substanciais na saúde. Para benefícios adicionais à saúde, os adultos devem aumentar a frequência semanal de exercícios para 300 minutos. Além 
disso, os adultos devem realizar atividades de fortalecimento muscular que envolvam os principais grupamentos musculares, duas vezes ou mais na semana, uma vez que tais atividades promovem benefícios adicionais à saúde. Muito embora, isso não permita garantir que indivíduos praticantes de uma ou até mesmo das três vertentes de atividades estejam imunes a qualquer tipo de doenças, sobretudo sobre o COVID-19, do qual ainda temos tão poucos estudos a respeito. Também é cedo para afirmar que os mesmos se recuperariam mais rapidamente, ou sequer teriam alguns sintomas ao praticar essas atividades - conforme orientações e recomendações dadas - caso fossem acometidos pelo referido vírus.

É certo que, com o isolamento social, são notórias as dificuldades da prática dos exercícios, uma vez que não é o melhor momento para incentivá-los ao ar livre, dificultando assim a prática de exercícios aeróbios como a corrida ou o ciclismo. Exceto as pessoas que possuem equipamentos ergométricos em domicílio. Em relação aos exercícios de força, existe a necessidade de adaptação, já que a falta de aparelhos de musculação exige substituição por exercícios que utilizam o peso do próprio corpo ou os pesos livres, já que a maioria não possui em sua residência aparelhos utilizados nas academias de ginástica. E quanto aos exercícios de alongamento/flexibilidade, talvez esses sejam o de melhor acessibilidade, mas assim como os demais, é preciso levar em consideração certos cuidados, no que diz respeito à segurança do praticante no momento de sua prática e realização, como: evitar exageros (na intensidade e/ou no volume), movimentos e posturas incorretas, mal posicionamento do corpo, atenção na respiração, velocidade da execução, impactos, desconfortos, aos intervalos de recuperação e/ou repouso, respeito aos próprios limites físicos e individualidades, nível a que se encontra o indivíduo, fases do treino (aquecimento, fase principal e resfriamento) e variabilidades (alternância nos tipos de treinos e exercícios).

Indivíduos considerados iniciantes, ou seja, com pouco tempo ou nenhuma prática em relação ao determinado treino, deverão realizar as atividades num esforço ou ritmo relativamente inferior ao intermediário e, principalmente, quando comparado àqueles já mais avançados na sua técnica de execução e condição física, cujo grupo deverá partir do pressuposto que deverão seguir os sinais e "avisos" subjetivos dados pelo próprio corpo, durante a prática, quanto ao nível de intensidade (Escala de Borg, Percepção Subjetiva de Esforço, entre outros métodos podem ser utilizados). Outra forma de perceber a intensidade do esforço é pela frequência respiratória, ${ }^{39}$ se determinado indivíduo não consegue mais conversar, por exemplo, provavelmente a intensidade estará elevada para o mesmo. Os praticantes considerados mais avançados, aqueles que já praticam por anos a modalidade em questão, de maneira ininterrupta, poderão realizar atividades de alto volume. Para o indivíduo em estágio mais avançado, apesar do esforço talvez significativamente mais intenso, o mesmo possui uma adaptação neuromuscular e cardiorrespiratória capaz de promover a sua recuperação de forma mais rápida em comparação ao iniciante, e ao mesmo tempo, pode-se entender que, ambos estarão recebendo, relativamente, 
um estímulo semelhante, mas cada um a seu nível de aptidão motora e física, não deixando de ser mais elevado por parte do primeiro. Mas, é importante ter uma rotina ou programação semanal, o que inclui o repouso e a recuperação necessários em decorrência do tipo de esforço e estímulo gerados ao organismo, para todos os casos e grupos - do iniciante ao avançado, e que certamente um profissional de Educação Física capacitado poderá auxiliar.

Portanto, é necessário equilibrar a frequência, o volume e a intensidade da prática de exercícios físicos a domicílio, durante esse período de isolamento. Nessa perspectiva, as pessoas precisam entender que não devem pensar em se tornar atletas nesse período. Engana-se achar que esse é o momento ideal para se buscar melhorar o desempenho atlético. Mas sim, diante desse cenário, enxergar nisso um importante aliado a fim de se manter ativo, respeitando os seus limites, particularidades, necessidades, expectativas, como já foi descrito, tendo em vista a prevenção de riscos e segurança acima de tudo, especialmente nesse momento delicado e imprevisível de que a população em geral está evidenciando e vivendo.

Ao realizar o mesmo exercício todos os dias, rotineiramente, se não controlar, $o$ indivíduo certamente estará aumentando o risco de uma lesão por esforço, justamente pelo fato de muitas vezes envolver os mesmos músculos e articulações. Todavia, se conseguir equilibrar a intensidade dos treinos regulares com a recuperação mais adequada aos tipos de treinos, certamente essa prática diária não trará qualquer problema, e muito ao contrário, acarretará benefícios. Nesse sentido, há a necessidade de alternar (intercalar) as atividades toda vez que for possível, ajustando de acordo com a disponibilidade de cada indivíduo, alternando o tipo de atividade com o descanso (intervalo para recuperação), para se adquirir melhores resultados e ao mesmo tempo não haver comprometimento de uma atividade para a outra, bem como, para evitar possíveis lesões que possam levar ao desinteresse e abandono à prática em domicílio. Por exemplo: Se um indivíduo for correr, não necessitará fazer isso todos os dias, poderá alternar a atividade durante a semana, com outro tipo já citado, como exercícios de força ou alongamentos. Como estão em quarentena, a mesma coisa para os exercícios realizados em domicílio (na maioria dos casos, com ênfase no treinamento de força). Nesse caso, uma ideia seria variar com exercícios de alongamento ou alternar o tipo de grupo muscular solicitado, por exemplo, realizar exercícios para membros inferiores num dia, e membros superiores e abdômen no outro.

E levando tudo isso em consideração, os profissionais de Educação Física, diante desse processo, têm uma grande e real responsabilidade, precisam ter em mente uma rápida classificação dos praticantes por níveis/grupos (iniciantes, intermediários e avançados); a intensidade do esforço e ritmo (baixa ou leve; média ou moderada; alta ou forte; muito alta ou muito forte); o volume e a duração do treinamento (entre 30 a 60 minutos); a frequência semanal dos treinos (três a cinco vezes, ao menos duas ou até mesmo diariamente), e a sua direta correlação com o tipo de atividade a ser realizada. Há importância de se adaptar e aderir aos recursos tecnológicos existentes como meio de estreitar a relação com o público-alvo e também com o público 
em geral, para que possam sair do sedentarismo, conscientizar-se da real importância da prática regular e adoção de novos hábitos. $\mathrm{E}$, ao público praticante de atividades físicas, cabe ressaltar que seria muito importante monitorar os "avisos" e "sinais" do organismo, afinal de contas, a saúde e o bem-estar devem ser mais importantes do que qualquer objetivo, seja ele qual for, condicionamento físico, emagrecimento, estética etc. Por sua vez, se o indivíduo apresentar algum problema de saúde, ou sintomas como de gripe, coriza, febre ou dor de cabeça e estiver indisposto a treinar, a recomendação seria focar a recuperação do corpo, permitindo alguns dias de descanso. Todavia, o importante é a regularidade e a manutenção das atividades, o que levará o sistema imune a estar mais fortalecido e saudável, com chances de combater mais eficientemente as inúmeras doenças e possivelmente os efeitos deletérios do $\mathrm{CO}$ VID-19.

\section{CONSIDERAÇões FINAIS}

Até o momento existem apenas algumas evidências de proteção do exercício físico em alguns tipos de vírus. Importante salientar que o COVID-19 é um vírus novo e não conhecemos totalmente a sua biologia. Como apresenta uma agressiva tempestade inflamatória, é difícil predizer se o efeito anti-inflamatório do exercício será impor- tante nesse desfecho. A resposta clínica do vírus varia individualmente de pessoa para pessoa, o que pode dificultar a interpretação da proteção do exercício físico. Também não existem estudos que demonstraram benefícios imunes dos exercícios físicos diretamente para proteção contra o COVID-19. Uma sugestão seria a utilização de modelos animais susceptíveis a esse novo vírus, algo extremamente complexo. Estudos experimentais em humanos também são um pouco limitantes, e certamente a aprovação destes pelos comitês de ética. Quanto às recomendações práticas, sugerimos realizar exercícios aeróbios, assim como os de força e alongamento. Importante também fazer os exercícios respeitando a capacidade física individual e evitar exceder 60 minutos de sessão intensa de exercício diário, para evitar imunossupressão neste momento de pandemia. Seguir também as recomendações do Colégio Americano de Medicina do Esporte (discutidas ao longo do texto), quanto à frequência semanal. A prática regular de exercícios físicos promoverá um bem-estar físico e mental importante para o enfrentamento durante o distanciamento social. Portanto, a partir desses estudos é possível afirmar que o exercício físico promove imunossegurança. No entanto, ainda é cedo para afirmar que um indivíduo ativo teria uma maior proteção contra o COVID-19. Aguardemos os estudos! 


\section{REFERÊNCIAS}

1. Jin Y., Yang H., Ji W., Wu W., Chen S, Zhang W., et al. Virology, epidemiology, pathogenesis, and control of covid-19. Viruses, 2020; 12 (4): E372.

2. Diagnosis and treatment protocol for novel coronavirus pneumonia; (Trial version 7). Chinese Med J. 2020; 133 (9) 1.087-1.095.

3. Fu Y., Cheng Y., Wu Y. Understanding SARS-Cov2-mediated inflammatory responses: from mechanisms to potential therapeutic tools. Virol Sin. 2020; 35: 266-271.

4. Huang C., Wang Y., Li X., Ren L., Zhao J., Hu Y., et al. Clinical features of patients infected with 2019 novel coronavirus in Wuhan, China. Lancet. 2020; 395 (10.223): 497-506.

5. Zhou P., Yang XL, Wang XG, Hu B., Zhang L., Zhang W, et al. A pneumonia outbreak associated with a new coronavirus of probable bat origin. Nature, 2020; 579 (7.798): 270-273.

6. Tvede N., Kappel M., Halkjaer-Kristensen J., Galbo H., Pedersen BK. The effect of light, moderate and severe bicycle exercise on lymphocytes subsets, natural and lymphokine activated killer cells, lymphocyte proliferative response and interleukin 2 production. Int J Sports Med.1993; 14 (5): 275-282.

7. Luci C., Tomasello E. Natural killer cells: detectors of stress. Int J Biochem Cell Biol. 2008; 40 (11): 2.335-2.340.

8. Shimasaki N., Jain A., Campana D. NK cells for cancer immunotherapy. Nat Rev Drug Discov 2020; 19 (3): 200-218.

9. Sellami M., Gasmi M., Denham J., Hayes LD, Stratton D., Padulo J., et al. Effects of acute and chronic exercise on immunological parameters in the elderly aged: can physical activity counteract the effects of aging? Front Immunol. 2018; 9: 2.187.

10. Nieman DC, Henson DA, Austin MD, Brown VA. Immune response to a 30-minute walk. Med Sci Sports Exerc. 2005; 37 (1): 57-62.

11. Adams GR, Zaldivar FP, Nance DM, Kodesh E., Radom-Aizik S., Cooper DM. Exercise and leukocyte interchange among central circulation, lung, spleen, and muscle. Brain Behav Immun. 2011; 25 (4): 658-666.

12. Simpson RJ, Kunz H., Agha N., Graff R. Exercise and the regulation of immune functions. Prog Mol Biol Transl Sci. 2015; 135: 355-380.

13. Turner JE, Spielmann G., Wadley AJ, Aldred S., Simpson RJ, Campbell JP. Exercise-induced B cell mobilization: preliminary evidence for an influx of immature cells into the bloodstream. Physiol Behav. 2016; 164: 376-382.

14. Northoff H., Berg A. Immunologic mediators as parameters of the reaction to strenuous exercise. Int J Sports Med. 1991; 12 (1): S9-S15.

15. Nieman DC, Buckley KS, Henson DA, Warren BJ, Suttles J., Ahle JC, Simandle S., et al. Immune function in marathon runners versus sedentary controls. Med Sci Sports Exerc. 1995; 27 (7): 986-992.

16. Nieman DC, Lila MA, Gillitt ND. Immunometabolism: a multi-omics approach to interpreting the influence of exercise and diet on the immune system. Ann Rev Food Sci Tech. 2019; 10: 341-363.

17. Simpson RJ, Campbell JP, Gleeson M., Kruger K., Nieman DC, Pyne DB, et al. Can exercise affect immune function to increase susceptibility to infection? Exerc Immunol Review. 2020; 26: 8-22.

18. Nieman DC, Johanssen LM, Lee JW, Arabatzis K. Infectious episodes in runners before and after the Los Angeles Marathon. J Sports Med Phys Fitness. 1990; 30 (3): 316-328.

19. Konig B. Psychological aspects of fighting stress: activity and relaxation must be well balanced. Plege Z.; 53 (11): 739-742. 
20. Nieman DC. Is infection risk linked to exercise workload? Med Sci Sports Exerc. 2000; 32 (7): S406-S411.

21. Soltani N., Marandi SM, Kazemi M., Esmaeil N. The exercise training modulatory effects on the obesity-induced immunometabolic dysfunctions. Diabetes Metab Syndr Obes. 2020; 13: 785810.

22. Duggal NA, Niemiro G., Harridge SDR, Simpson RJ, Lord JM. Can physical activity ameliorate immunosenescence and thereby reduce age-related multi-morbidity? Nat Rev Immunol. 2019; 19 (9): 563-572.

23. Pedersen BK. Anti-inflammatory effects of exercise: role in diabetes and cardiovascular disease. Eur J Clin Invest. 2017; 47 (8): 600-611.

24. Wang B., Li R., Lu Z., Huang Y. Does comorbidity increase the risk of patients with Covid-19: evidence from meta-analysis. Aging. 2020; 12 (7): 6.049-6.057.

25. Nikolich-Zugich J., Knox KS, Rios CT, Natt B., Bhattacharya D., Fain MJ. SARS-Cov2 and COVID-19 in older adults: what we may expect regarding pathogenesis, immune responses, and outcomes. Geroscience. 2020, 42 (3): 1.013.

26. Wu S., Ma C., Yang Z., Yang P., Chu Y., Zhang H., et al. Hygiene behaviors associated with influenza-like illness among adults in Beijing, China: a large, population-based survey. PLoS One. 2016; 11 (2): e 0148448.

27. Wong CM, Lai HK, Ou CK, Ho SY, Chan KP, Thach TQ, et al. Is exercise protective against influenza-associated mortality? PLoS One. 2008; 3 (5): e 2.108.

28. Lowder T., Padgett DA, Woods JA. Moderate exercise protects mice from death due to influenza vírus. Brain Behav Immun. 2005; 19 (5): 377-380.

29. Woods JA, Keylock K, Lowder T., Vieira VJ, Zelkovich W., Dumich S., et al. Cardiovascular exercise training extends influenza vaccine seroprotection in sedentary older adults: immune function intervention trial. J Am Geriatr Soc. 2009; 57 (12): 2.183-2.191.

30. Agha NH, Mehta SK, Rooney BV, Laughlin MS, Markofski MM, Pierson DL, et al. Exercise as a countermeasure for latent viral reactivation during long duration space flight. FASEB J. 2020; 34 (2): 2.869-2.881.

31. Emery CF, Kiecolt-Glaser JK, Glaser R., Malarkey WB, Frid DJ. Exercise accelerates wound healing among healthy older adults: a preliminary investigation. J Gerontol a Biol Sci Med Sci 2005; 60 (11): 1.432-1.436.

32. Pedersen BK. Which type of exercise keeps you young? Curr Opin Clin Nutr Metab Care. 2019; 22 (2): 167-173.

33. Cox MH. Exercise training programs and cardiorespiratory adaptation. Clin Sports Med. 1991; 10 (1): 19-32.

34. Hunter GR, Byrne NM, Gower BA, Sirikul B., Hills AP. Increased resting energy expenditure after 40 minutes of aerobic but not resistance exercise. Obesity. 2006; 14 (11): 2.018-2.025.

35. Deschenes MR, Kraemer WJ. Performance and physiologic adaptations to resistance training. Am J Phys Med Rehabil. 2002; 81 (11): 3-16.

36. Phillips SM. Resistance exercise: good for more than just Grandma and Grandpa's muscles. Appl Physiol Nutr Metab. 2007; 32 (6): 1.198-1.205.

37. Guissard N., Duchateau J. Neural aspects of muscle stretching. Exerc Sport Sci Rev. 2006; 34 (4): 154-158.

38. Garber CE, Blissmer B., Dechenes MR, Franklin BA, Lamonte MJ, Lee I-Min, et al. American College of Sports Medicine Position Stand. Quantity and quality of exercise for developing and maintaining cardiorespiratory, musculoskeletal, and neuromotor fitness in apparently healthy adults: guidance for prescribing exercise. Med Sci Sports Exerc 2011; 43 (7): 1.334-1.359. 
39. Nicolò A., Massaroni C., Passfield L. Respiratory frequency during exercise: the neglected physiological measure. Front Physiol. 2017; 8: 922.

\section{DADOS DOS AUTORES}

\section{André Katayama Yamada}

Doutor em Ciências do Movimento Humano pela Universidade Metodista de Piracicaba (UNIMEP). Piracicaba/SP - Brasil. yamadaak@gmail.com

\section{Luiz Otávio Barreto Polis}

Pós graduado (especialização) pelo Centro Universitário Internacional (UNINTER). Piracicaba/SP

- Brasil.lopolis_tavinho@hotmail.com

Submetido em: $15-7-2020$

Aceito em: 1-8-2020 